\title{
ON AN INTEGRAL OPERATOR
}

\section{Virgil PESCAR and Daniel BREAZ}

\begin{abstract}
In this paper we define a general integral operator for analytic functions in the open unit disk and we determine some conditions for univalence of this integral operator.
\end{abstract}

\section{Introduction}

Let $\mathcal{A}$ be the class of functions $f$ of the form

$$
f(z)=z+\sum_{n=2}^{\infty} a_{n} z^{n}
$$

normalized by $f(0)=f^{\prime}(0)-1=0$, which are analytic in the open unit disk $\mathcal{U}=\{z \in \mathbb{C}:|z|<1\}$.

We consider $\mathcal{S}$ the subclass of $\mathcal{A}$ consisting of functions $f \in \mathcal{A}$, which are univalent in $\mathcal{U}$.

We denote by $\mathcal{P}$ the class of functions $p$ of the form

$$
p(z)=1+\sum_{k=1}^{\infty} b_{k} z^{k}
$$

which are analytic in $\mathcal{U}$, with $\operatorname{Re} p(z)>0$, for all $z \in \mathcal{U}$.

In this work we introduce a new integral operator defined by

$$
V_{n}(z)=\left\{\beta \int_{0}^{z} u^{\beta-1}\left(p_{1}(u)\right)^{\gamma_{1}} \ldots\left(p_{n}(u)\right)^{\gamma_{n}} d u\right\}^{\frac{1}{\beta}}
$$

for functions $p_{j} \in \mathcal{P}$ and $\beta, \gamma_{j}$ be complex numbers $\beta \neq 0$ and $j=\overline{1, n}$.

Key Words: Analytic, integral operator, univalence.

2010 Mathematics Subject Classification: Primary 30C45.

Received: May, 2013.

Revised: October, 2013

Accepted: November, 2013. 


\section{Preliminary results}

We shall use the following lemmas.

Lemma 2.1. [3]. Let $\alpha$ be a complex number, Re $\alpha>0$ and $f \in \mathcal{A}$. If

$$
\frac{1-|z|^{2 \operatorname{Re} \alpha}}{\operatorname{Re} \alpha}\left|\frac{z f^{\prime \prime}(z)}{f^{\prime}(z)}\right| \leq 1
$$

for all $z \in \mathcal{U}$, then for any complex number $\beta, \operatorname{Re} \beta \geq \operatorname{Re} \alpha$, the function

$$
F_{\beta}(z)=\left[\beta \int_{0}^{z} u^{\beta-1} f^{\prime}(u)\right]^{\frac{1}{\beta}}
$$

is regular and univalent in $\mathrm{U}$.

Lemma 2.2. (Schwarz [1]). Let $f$ be the function regular in the disk

$\mathcal{U}_{R}=\{z \in \mathbb{C}:|z|<R\}$, with $|f(z)|<M, M$ fixed. If $f(z)$ has in $z=0$ one zero with multiply $\geq m$, then

$$
|f(z)| \leq \frac{M}{R^{m}}|z|^{m},\left(z \in \mathcal{U}_{R}\right),
$$

the equality (in the inequality (3) for $z \neq 0$ ) can hold only if

$$
f(z)=e^{i \theta} \frac{M}{R^{m}} z^{m}
$$

where $\theta$ is constant.

Lemma 2.3. [2]. If the function $f$ is regular in $\mathcal{U}$ and $|f(z)|<1$ in $\mathcal{U}$, then for all $\xi \in \mathcal{U}$ and $z \in \mathcal{U}$ the following inequalities hold

$$
\begin{gathered}
\left|\frac{f(\xi)-f(z)}{1-\overline{f(z)} f(\xi)}\right| \leq \frac{|\xi-z|}{|1-\bar{z} \xi|}, \\
\left|f^{\prime}(z)\right| \leq \frac{1-|f(z)|^{2}}{1-|z|^{2}}
\end{gathered}
$$

the equalities hold only in the case $f(z)=\frac{\epsilon(z+u)}{1+\bar{u} z}$, where $|\epsilon|=1$ and $|u|<1$.

Remark 2.4. [2]. For $z=0$, from inequality (4)

$$
\left|\frac{f(\xi)-f(0)}{1-\overline{f(0)} f(\xi)}\right| \leq|\xi|
$$


and, hence

$$
|f(\xi)| \leq \frac{|\xi|+|f(0)|}{1+|f(0)||\xi|}
$$

Considering $f(0)=a$ and $\xi=z$, we have

$$
|f(z)| \leq \frac{|z|+|a|}{1+|a||z|}
$$

for all $z \in \mathcal{U}$.

\section{Main results}

Theorem 3.1. Let $\alpha, \beta, \gamma_{j}$ be complex numbers, $j=\overline{1, n}$, Re $\alpha>0$ and $p_{j} \in \mathcal{P}, p_{j}(z)=1+b_{1 j} z+b_{2 j} z^{2}+\ldots, j=\overline{1, n}$.

$$
\text { If }
$$

$$
\left|\frac{z p_{j}^{\prime}(z)}{p_{j}(z)}\right| \leq \frac{(2 R e \alpha+1)^{\frac{2 R e \alpha+1}{2 R e} \alpha}}{2}, \quad(z \in \mathcal{U} ; j=\overline{1, n})
$$

and

$$
\left|\gamma_{1}\right|+\left|\gamma_{2}\right|+\ldots+\left|\gamma_{n}\right| \leq 1
$$

then for any complex number $\beta$, Re $\beta \geq \operatorname{Re} \alpha$, the integral operator $V_{n}$ defined by (1) is in the class $\mathcal{S}$.

Proof. Let's consider the function

$$
g_{n}(z)=\int_{0}^{z}\left(p_{1}(u)\right)^{\gamma_{1}} \ldots\left(p_{n}(u)\right)^{\gamma_{n}} d u, \quad\left(p_{j} \in \mathcal{P} ; j=\overline{1, n}\right) .
$$

The function $g_{n}$ is regular in $\mathcal{U}$ and $g_{n}(0)=g_{n}^{\prime}(0)-1=0$.

From (3) we obtain

$$
\frac{1-|z|^{2 \operatorname{Re} \alpha}}{\operatorname{Re} \alpha}\left|\frac{z g_{n}^{\prime \prime}(z)}{g_{n}^{\prime}(z)}\right| \leq \frac{1-|z|^{2 \operatorname{Re} \alpha}}{\operatorname{Re} \alpha} \sum_{j=1}^{n}\left|\gamma_{j}\right|\left|\frac{z p_{j}^{\prime}(z)}{p_{j}(z)}\right|,
$$

for all $z \in \mathcal{U}$.

By (1), applying Lemma 2.2 we have

$$
\left|\frac{z p_{j}^{\prime}(z)}{p_{j}(z)}\right| \leq \frac{(2 R e \alpha+1)^{\frac{2 R e \alpha+1}{2 R e} \alpha}}{2}|z|, \quad(z \in \mathcal{U} ; j=\overline{1, n}),
$$


and hence, by (4) we get

$$
\frac{1-|z|^{2 \operatorname{Re} \alpha}}{\operatorname{Re} \alpha}\left|\frac{z g_{n}^{\prime \prime}(z)}{g_{n}^{\prime}(z)}\right| \leq \frac{1-|z|^{2 \operatorname{Re} \alpha}}{\operatorname{Re} \alpha}|z| \frac{(2 \operatorname{Re} \alpha+1)^{\frac{2 \operatorname{Re} \alpha+1}{2 \operatorname{Re} \alpha}}}{2} \sum_{j=1}^{n}\left|\gamma_{j}\right|
$$

for all $z \in \mathcal{U}$.

Since

$$
\max _{|z| \leq 1}\left[\frac{1-|z|^{2 \operatorname{Re} \alpha}}{\operatorname{Re} \alpha}|z|\right]=\frac{2}{(2 \operatorname{Re} \alpha+1)^{\frac{2 \operatorname{Re} \alpha+1}{2 \operatorname{Re} \alpha}}},
$$

from (2) and (6), we obtain

$$
\frac{1-|z|^{2 \operatorname{Re} \alpha}}{\operatorname{Re} \alpha}\left|\frac{z g_{n}^{\prime \prime}(z)}{g_{n}^{\prime}(z)}\right| \leq 1, \quad(z \in \mathcal{U}) .
$$

From $(7)$ and since $g_{n}^{\prime}(z)=\left(p_{1}(z)\right)^{\gamma_{1}} \ldots\left(p_{z}(u)\right)^{\gamma_{n}}$, by Lemma 2.1 it results that the integral operator $V_{n}$ defined by (1) is in the class $\mathcal{S}$.

Theorem 3.2. Let $\alpha, \beta, \gamma_{j}$ be complex numbers, $j=\overline{1, n}$, Re $\alpha>0$, $M_{j}$ positive real numbers and $p_{j} \in \mathcal{P}, p_{j}(z)=1+b_{1 j} z+b_{2 j} z^{2}+\ldots, j=\overline{1, n}$.

If

$$
\begin{gathered}
\left|\frac{p_{j}^{\prime}(z)}{p_{j}(z)}\right|<M_{j}, \quad(z \in \mathcal{U} ; j=\overline{1, n}), \\
M_{1}\left|\gamma_{1}\right|+M_{2}\left|\gamma_{2}\right|+\ldots+M_{n}\left|\gamma_{n}\right| \leq \frac{1}{\max _{|z| \leq 1}\left[\frac{1-|z|^{2 \operatorname{Re} \alpha}}{\operatorname{Re} \alpha}|z| \frac{|z|+|c|}{1+|c||z|}\right]},
\end{gathered}
$$

where

$$
c=\frac{b_{11} \gamma_{1}+b_{12} \gamma_{2}+\ldots+b_{1 n} \gamma_{n}}{M_{1}\left|\gamma_{1}\right|+M_{2}\left|\gamma_{2}\right|+\ldots+M_{n}\left|\gamma_{n}\right|}
$$

then for any complex number $\beta, \operatorname{Re} \beta \geq \operatorname{Re} \alpha$, the integral operator $V_{n}$ given by (1) is in the class $\mathcal{S}$.

Proof. We consider the function

$$
g_{n}(z)=\int_{0}^{z}\left(p_{1}(u)\right)^{\gamma_{1}} \ldots\left(p_{n}(u)\right)^{\gamma_{n}} d u
$$

which is regular in $\mathcal{U}$ and $g_{n}(0)=g_{n}^{\prime}(0)-1=0$. 
Let's consider the function

$$
h(z)=\frac{1}{M_{1}\left|\gamma_{1}\right|+M_{2}\left|\gamma_{2}\right|+\ldots+M_{n}\left|\gamma_{n}\right|} \frac{g_{n}^{\prime \prime}(z)}{g_{n}^{\prime}(z)}, \quad(z \in \mathcal{U})
$$

and from (11) we get

$$
\begin{aligned}
h(z) & =\frac{\gamma_{1}}{M_{1}\left|\gamma_{1}\right|+\ldots+M_{n}\left|\gamma_{2}\right|} \frac{p_{1}^{\prime}(z)}{p_{1}(z)}+\ldots+ \\
& +\frac{\gamma_{n}}{M_{1}\left|\gamma_{1}\right|+\ldots+M_{n}\left|\gamma_{2}\right|} \frac{p_{n}^{\prime}(z)}{p_{n}(z)},
\end{aligned}
$$

for all $z \in \mathcal{U}$.

From (8) and (13) we obtain $|h(z)|<1, z \in \mathcal{U}$.

We have

$$
h(0)=\frac{b_{11} \gamma_{1}+b_{12} \gamma_{2}+\ldots+b_{1 n} \gamma_{n}}{M_{1}\left|\gamma_{1}\right|+M_{2}\left|\gamma_{2}\right|+\ldots+M_{n}\left|\gamma_{n}\right|}=c
$$

and by applying the Remark 2.4 we get

$$
|h(z)| \leq \frac{|z|+|c|}{1+|c||z|}, \quad(z \in \mathcal{U}),
$$

where

$$
|c|=\frac{\left|b_{11} \gamma_{1}+b_{12} \gamma_{2}+\ldots+b_{1 n} \gamma_{n}\right|}{M_{1}\left|\gamma_{1}\right|+M_{2}\left|\gamma_{2}\right|+\ldots+M_{n}\left|\gamma_{n}\right|},
$$

From (12), (14) we obtain

$$
\begin{gathered}
\frac{1-|z|^{2 \operatorname{Re} \alpha}}{\operatorname{Re} \alpha}\left|\frac{z g_{n}^{\prime \prime}(z)}{g_{n}^{\prime}(z)}\right| \leq \\
\leq\left(M_{1}\left|\gamma_{1}\right|+\ldots+M_{n}\left|\gamma_{n}\right|\right) \max _{|z| \leq 1}\left[\frac{1-|z|^{2 \operatorname{Re} \alpha}}{\operatorname{Re} \alpha}|z| \frac{|z|+|c|}{1+|c||z|}\right]
\end{gathered}
$$

for all $z \in \mathcal{U}$.

From (9) and (15) we have

$$
\frac{1-|z|^{2 \operatorname{Re} \alpha}}{\operatorname{Re} \alpha}\left|\frac{z g_{n}^{\prime \prime}(z)}{g_{n}^{\prime}(z)}\right| \leq 1, \quad(z \in \mathcal{U}) .
$$

From (16) and since $g_{n}^{\prime}(z)=\left(p_{1}(z)\right)^{\gamma_{1}} \ldots\left(p_{n}(z)\right)^{\gamma_{n}}$, by Lemma 2.1, it results that the integral operator $V_{n}$ given by (1) belongs to the class $\mathcal{S}$. 
Theorem 3.3. Let $\alpha, \gamma_{j}$ be complex numbers, $j=\overline{1, n}$, Re $\alpha>0$ and $p_{j} \in \mathcal{P}$, $p_{j}(z)=1+b_{1 j}(z)+b_{2 j}(z)+\ldots, j=\overline{1, n}$.

If

$$
\left|\gamma_{1}\right|+\left|\gamma_{2}\right|+\ldots+\left|\gamma_{n}\right| \leq \frac{\operatorname{Re} \alpha}{2}, \quad(0<\operatorname{Re} \alpha \leq 1)
$$

or

$$
\left|\gamma_{1}\right|+\left|\gamma_{2}\right|+\ldots+\left|\gamma_{n}\right| \leq \frac{1}{2}, \quad(\operatorname{Re} \alpha>1),
$$

then for any complex number $\beta$, Re $\beta \geq$ Re $\alpha$, the integral operator $V_{n} \in \mathcal{S}$.

Proof. Since $p_{j} \in \mathcal{P}, j=\overline{1, n}$, we have

$$
\left|\frac{z p_{j}^{\prime}(z)}{p_{j}(z)}\right| \leq \frac{2|z|}{1-|z|^{2}}, \quad(z \in \mathcal{U}) .
$$

We consider the function

$$
g_{n}(z)=\int_{0}^{z}\left(p_{1}(u)\right)^{\gamma_{1}} \ldots\left(p_{n}(u)\right)^{\gamma_{n}} d u
$$

From (19) and (20) we obtain

$$
\begin{gathered}
\frac{1-|z|^{2 \operatorname{Re} \alpha}}{\operatorname{Re} \alpha}\left|\frac{z g_{n}^{\prime \prime}(z)}{g_{n}^{\prime}(z)}\right| \leq \\
\leq \frac{1-|z|^{2 \operatorname{Re} \alpha}}{\operatorname{Re} \alpha} \cdot \frac{2|z|}{1-|z|^{2}} \sum_{j=1}^{n}\left|\gamma_{j}\right|, \quad(z \in \mathcal{U}) .
\end{gathered}
$$

For $0<\operatorname{Re} \alpha \leq 1$ we have $1-|z|^{2 R e} \alpha \leq 1-|z|^{2}$, for all $z \in \mathcal{U}$. By (17) and (21) we get

$$
\frac{1-|z|^{2 \operatorname{Re} \alpha}}{\operatorname{Re} \alpha}\left|\frac{z g_{n}^{\prime \prime}(z)}{g_{n}^{\prime}(z)}\right| \leq 1, \quad(z \in \mathcal{U} ; 0<\operatorname{Re} \alpha \leq 1) .
$$

For Re $\alpha>1$ we obtain $\frac{1-|z|^{2 R e} \alpha}{\operatorname{Re} \alpha} \leq 1-|z|^{2}$, for all $z \in \mathcal{U}$. By (18) and (21) we get

$$
\frac{1-|z|^{2 \operatorname{Re} \alpha}}{\operatorname{Re} \alpha}\left|\frac{z g_{n}^{\prime \prime}(z)}{g_{n}^{\prime}(z)}\right| \leq 1, \quad(z \in \mathfrak{U} ; \operatorname{Re} \alpha>1) .
$$

From (22), (23) and Lemma 2.1 we obtain that $V_{n} \in \mathcal{S}$. 


\section{Corollaries}

Corollary 4.1. Let $\alpha, \gamma_{j}$ be complex numbers, $j=\overline{1, n} 0<$ Re $\alpha \leq 1$ and $p_{j} \in \mathcal{P}, p_{j}(z)=1+b_{1 j}(z)+b_{2 j}(z)+\ldots, j=\overline{1, n}$.

If

$$
\begin{gathered}
\left|\frac{z p_{j}^{\prime}(z)}{p_{j}(z)}\right| \leq \\
\qquad \frac{(2 \operatorname{Re} \alpha+1)^{\frac{2 R e \alpha+1}{2 R e} \alpha}}{2}, \quad(z \in \mathcal{U} ; j=\overline{1, n}), \\
\left|\gamma_{1}\right|+\left|\gamma_{2}\right|+\ldots+\left|\gamma_{n}\right| \leq 1,
\end{gathered}
$$

then the integral operator defined by

$$
K_{n}(z)=\int_{0}^{z}\left(p_{1}(u)\right)^{\gamma_{1}} \ldots\left(p_{n}(u)\right)^{\gamma_{n}} d u
$$

is in the class $\mathcal{S}$.

Proof. For $\beta=1$, from Theorem 3.1, we obtain the Corollary 4.1.

Corollary 4.2. Let $\alpha, \gamma_{j}$ be complex numbers, $j=\overline{1, n}, 0<$ Re $\alpha \leq 1, M_{j}$ positive real numbers and $p_{j} \in \mathcal{P}, p_{j}(z)=1+b_{1 j} z+b_{2 j} z^{2}+\ldots, j=\overline{1, n}$.

$$
\text { If }
$$

$$
\begin{gathered}
\left|\frac{p_{j}^{\prime}(z)}{p_{j}(z)}\right| \leq M_{j}, \quad(z \in \mathcal{U} ; j=\overline{1, n}), \\
M_{1}\left|\gamma_{1}\right|+M_{2}\left|\gamma_{2}\right|+\ldots+M_{n}\left|\gamma_{n}\right| \leq \frac{1}{\max _{|z| \leq 1}\left[\frac{1-|z|^{2 R e} \alpha}{R e \alpha}|z| \frac{|z|+|c|}{1+|c||z|}\right]},
\end{gathered}
$$

where

$$
c=\frac{b_{11} \gamma_{1}+b_{12} \gamma_{2}+\ldots+b_{1 n} \gamma_{n}}{M_{1}\left|\gamma_{1}\right|+M_{2}\left|\gamma_{2}\right|+\ldots+M_{n}\left|\gamma_{n}\right|}
$$

then the integral operator $K_{n}$ defined by (3) belongs to the class $\mathcal{S}$.

Proof. We take $\beta=1$ in Theorem 3.2.

Corollary 4.3. Let $\alpha, \beta, \gamma_{j}$ be complex numbers, $j=\overline{1, n}$, Re $\beta \geq \operatorname{Re} \alpha>0$, $M_{j}$ positive real numbers and $p_{j} \in \mathcal{P}, p_{j}(z)=1+b_{1 j} z+b_{2 j} z^{2}+\ldots, j=\overline{1, n}$. 
If

$$
\begin{gathered}
\left|\frac{p_{j}^{\prime}(z)}{p_{j}(z)}\right| \leq M_{j}, \quad(z \in \mathcal{U}, j=\overline{1, n}), \\
\left|b_{11} \gamma_{1}+b_{12} \gamma_{2}+\ldots+b_{1 n} \gamma_{n}\right| \leq \frac{(2 \operatorname{Re} \alpha+1)^{\frac{2 R e \alpha+1}{2 R e} \alpha}}{2} \\
\left|b_{11} \gamma_{1}+b_{12} \gamma_{2}+\ldots+b_{1 n} \gamma_{n}\right|=M_{1}\left|\gamma_{1}\right|+M_{2}\left|\gamma_{2}\right|+\ldots+M_{n}\left|\gamma_{n}\right|,
\end{gathered}
$$

then the integral operator $V_{n} \in \mathcal{S}$.

Proof. By (10), from Theorem 3.2 and (9) we have $|c|=1$.

Using the inequality (9) we obtain

$$
M_{1}\left|\gamma_{1}\right|+M_{2}\left|\gamma_{2}\right|+\ldots+M_{n}\left|\gamma_{n}\right| \leq \frac{1}{\max _{|z| \leq 1} \frac{1-|z|^{2 R e} \alpha}{\operatorname{Re} \alpha}|z|} .
$$

We have

$$
\max _{|z| \leq 1}\left[\frac{1-|z|^{2 \operatorname{Re} \alpha}}{\operatorname{Re} \alpha}|z|\right]=\frac{2}{(2 \operatorname{Re} \alpha+1)^{\frac{2 R e}{2 \operatorname{Re} \alpha} \alpha}}
$$

and from (9), (10) and (11) we obtain (8). The conditions of Theorem 3.2 are satisfied.

Corollary 4.4. Let $\alpha, \beta, \gamma_{j}$ be complex numbers, $j=\overline{1, n}$, Re $\beta \geq \operatorname{Re} \alpha>0$, $M_{j}$ positive real numbers and $p_{j} \in \mathcal{P}, p_{j}(z)=1+b_{1 j} z+b_{2 j} z^{2}+\ldots, j=\overline{1, n}$, $b_{11} \gamma_{1}+b_{12} \gamma_{2}+\ldots+b_{1 n} \gamma_{n}=0$.

If

$$
\begin{gathered}
\left|\frac{p_{j}^{\prime}(z)}{p_{j}(z)}\right| \leq M_{j}, \quad(z \in \mathcal{U}, j=\overline{1, n}), \\
M_{1}\left|\gamma_{1}\right|+M_{2}\left|\gamma_{2}\right|+\ldots+M_{n}\left|\gamma_{n}\right| \leq(\operatorname{Re} \alpha+1)^{\frac{\operatorname{Re} \alpha+1}{\operatorname{Re} \alpha}},
\end{gathered}
$$

then the integral operator $V_{n} \in \mathcal{S}$.

Proof. From (10) we have $c=0$ and using the inequality (9) we obtain

$$
M_{1}\left|\gamma_{1}\right|+M_{2}\left|\gamma_{2}\right|+\ldots+M_{n}\left|\gamma_{n}\right| \leq \frac{1}{\max _{|z| \leq 1} \frac{1-|z|^{2 R e} \alpha}{R e \alpha}|z|^{2}} .
$$


Since

$$
\max _{|z| \leq 1}\left[\frac{1-|z|^{2 \operatorname{Re} \alpha}}{\operatorname{Re} \alpha}|z|^{2}\right]=\frac{1}{(\operatorname{Re} \alpha+1)^{\frac{\operatorname{Re} \alpha+1}{\operatorname{Re} \alpha}}}
$$

from (14) we have the inequality (13).

The conditions of Theorem 3.2 are verified and hence, we obtain $V_{n} \in \mathcal{S}$.

Corollary 4.5. Let $\alpha, \beta, \gamma_{j}$ be complex numbers, $j=\overline{1, n}, 0<$ Re $\alpha \leq 1$ and $p_{j} \in \mathcal{P}, p_{j}(z)=1+b_{1 j} z+b_{2 j} z^{2}+\ldots, j=\overline{1, n}$.

If

$$
\left|\gamma_{1}\right|+\left|\gamma_{2}\right|+\ldots+\left|\gamma_{n}\right| \leq \frac{\operatorname{Re} \alpha}{2}, \quad(0<\operatorname{Re} \alpha \leq 1),
$$

then the integral operator $K_{n}$ given by (3) is in the class $\mathcal{S}$.

Proof. We take $\beta=1$ in Theorem 3.3.

\section{References}

[1] Mayer, O., The Functions Theory of One Variable Complex, Bucureşti, 1981.

[2] Nehari, Z., Conformal Mapping, Mc Graw-Hill Book Comp., New York, 1952 (Dover. Publ. Inc., 1975).

[3] Pascu, N. N., An improvement of Becker's univalence criterion, Proceedings of the Commemorative Session Simion Stoilow (Braşov), Preprint (1987), 43-48.

Virgil PESCAR,

Department of Mathematics,

"Transilvania" University of Braşov,

Faculty of Mathematics and Computer Science, 500091 Braşov, Romania

Email: virgilpescar@unitbv.ro

Daniel BREAZ,

Department of Mathematics,

"1 Decembrie 1918" University of Alba Iulia,

Faculty of Science, 510009 Alba Iulia, Romania.

Email: dbreaz@uab.ro 
